\title{
Fabrication of High-Density and Superuniform Gold Nanoelectrode Arrays for Electrochemical Fluorescence Imaging
}

Xiang Qin, Zhong-Qiu Li, Yue Zhou, Jian-Bin Pan, Jian Li, Kang Wang, Jing-Juan Xu and Xing-Hua Xia*

State Key Lab of Analytical Chemistry for Life Science, School of Chemistry and Chemical Engineering, Nanjing University, Nanjing 210023, China.

\section{Table of Contents}

Figure S1. Schematic illustration of the fabrication process of gold nanoelectrode arrays $-\mathrm{S}-2$

Figure S2. Photo images of the AAO membranes after electrodeposition. $-\mathrm{S}-2$

Figure S3. AFM images of the gold nanoelectrode arrays after polishing. $-\mathrm{S}-3$

Figure S4. Fluorescence on a pure gold plate bipolar electrode. S-4

Figure S5. Confocal images of the nanoelectrode arrays. S-5 


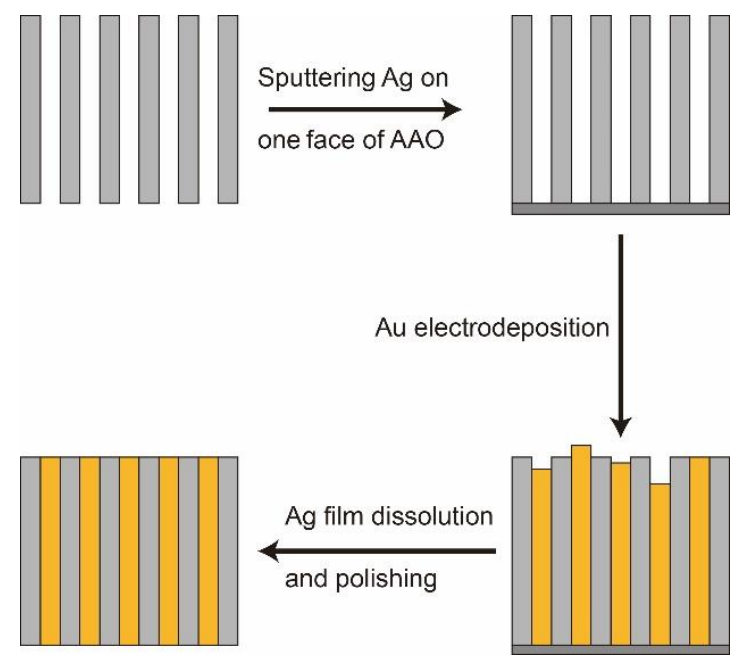

Figure S1. Schematic illustration of the fabrication process of gold nanoelectrode arrays.

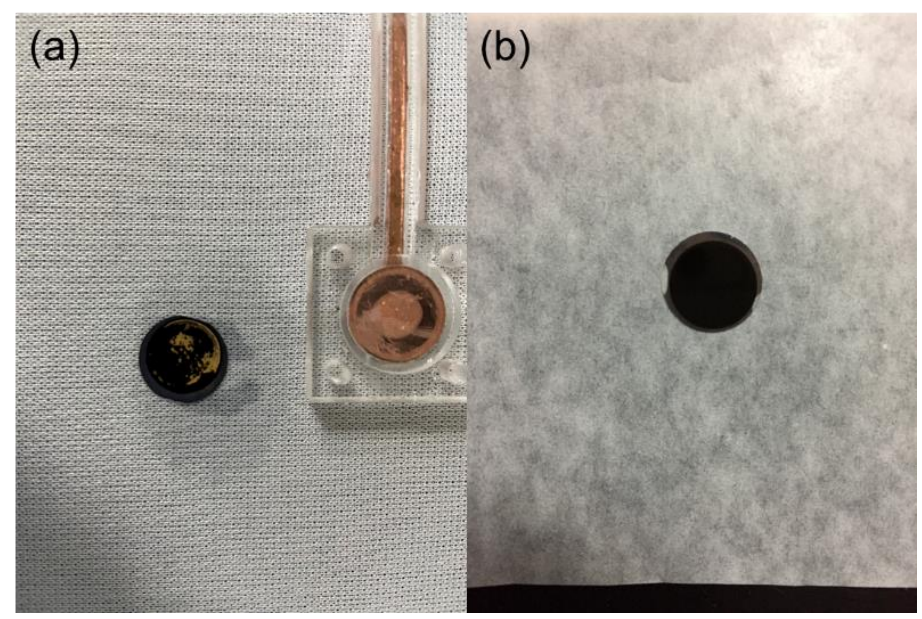

Figure S2. (a) Photo images of the AAO membrane with $140 \mathrm{~nm}$ pore diameter and $450 \mathrm{~nm}$ pore pitch after electrodeposition. The golden region indicates the overgrown gold layer. (b) Photo image of the gold nanoelectrode array after mechanical polishing. 

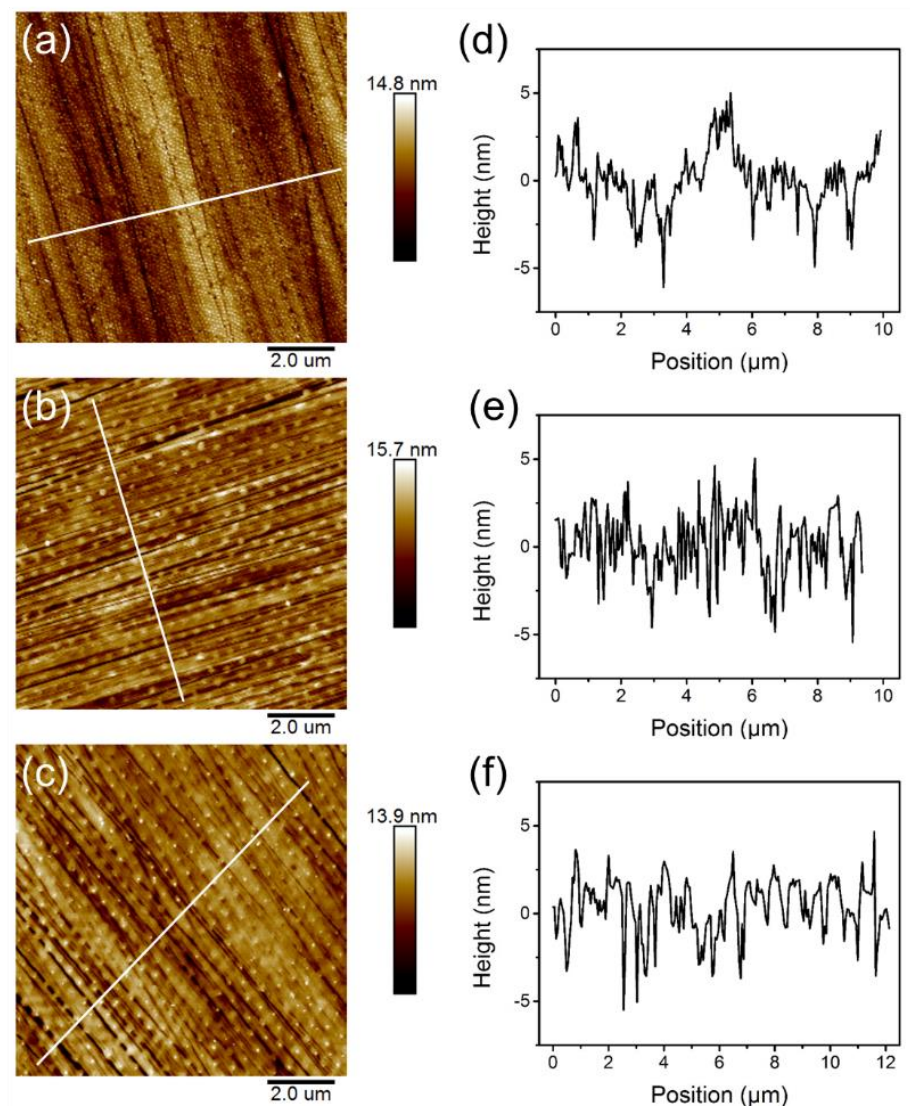

Figure S3. (a-c) AFM images of the gold nanoelectrode arrays with $60 \mathrm{~nm}$ electrode diameter and $125 \mathrm{~nm}$ electrode pitch (a), $140 \mathrm{~nm}$ electrode diameter and $450 \mathrm{~nm}$ electrode pitch (b), $200 \mathrm{~nm}$ electrode diameter and $450 \mathrm{~nm}$ electrode pitch (c). (d-f) Profiles of surface height of the white lines in (a), (b) and (c), respectively. 

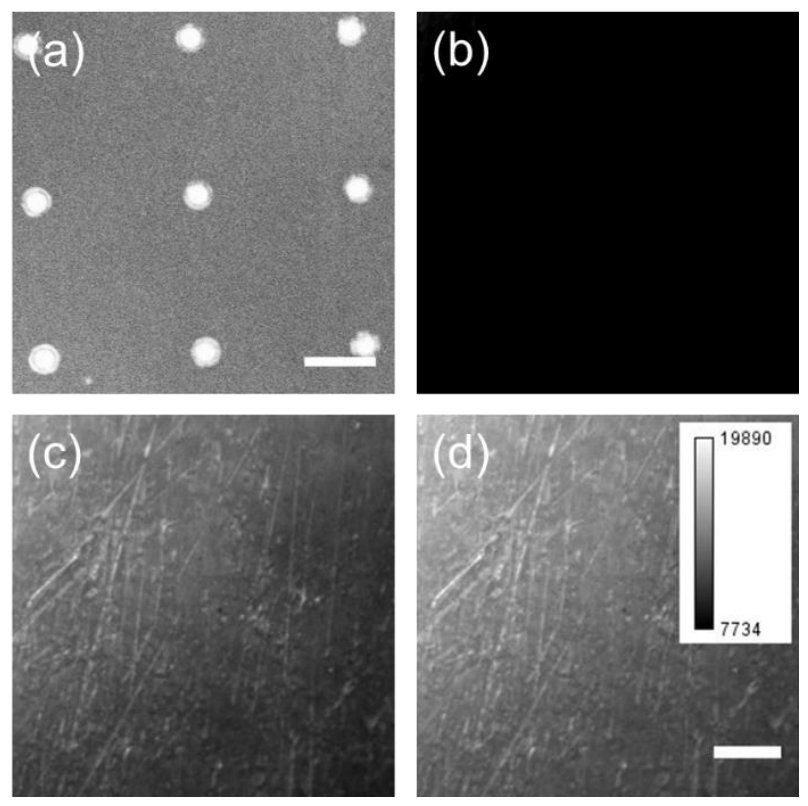

Figure S4. (a) SEM image of the microwells fabricated on one side of a pure gold plate. (b-d) Three fluorescence snapshots extracted from a video recorded at the anodic side of the gold plate bipolar electrode. All sale bars are $50 \mu \mathrm{m}$. 


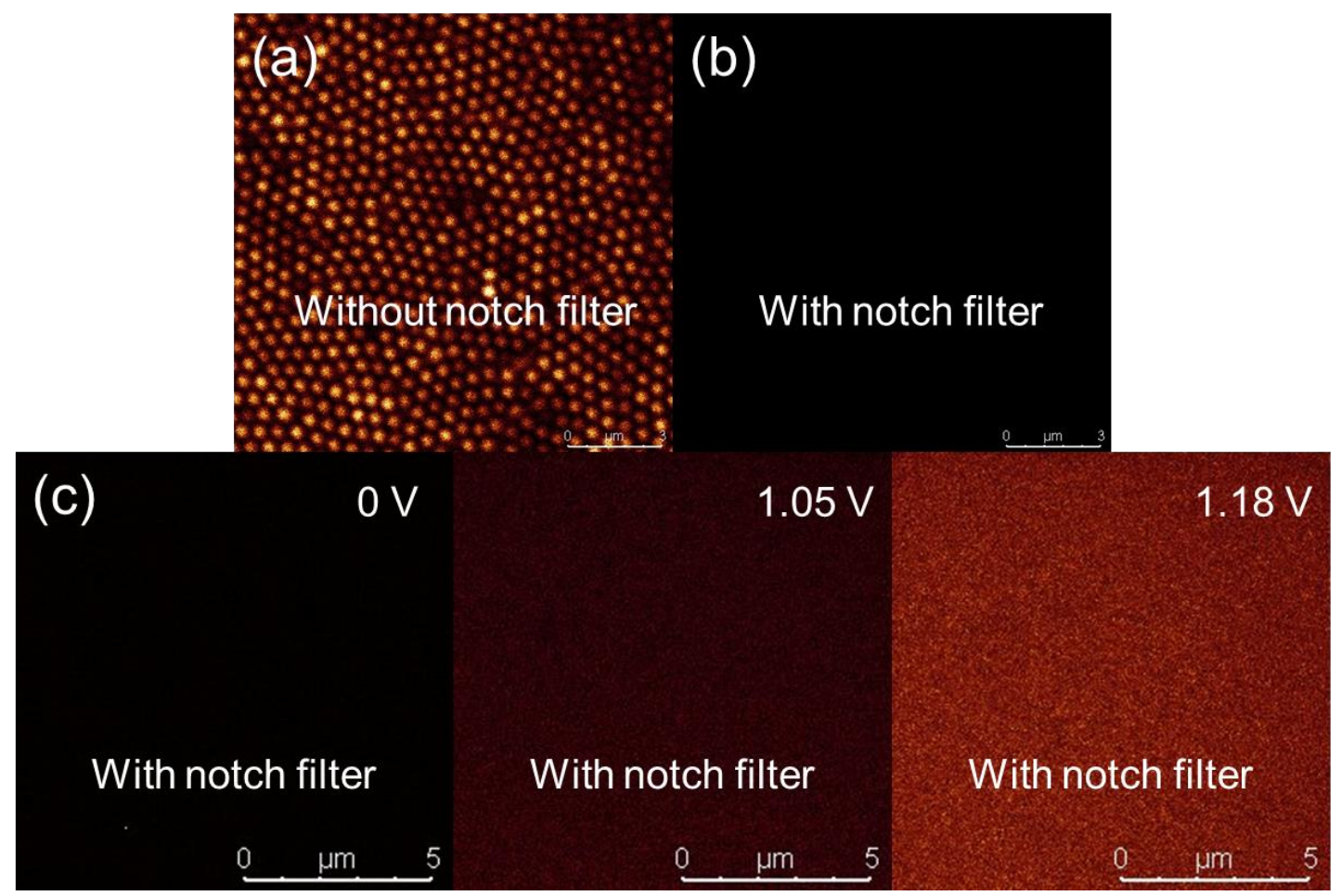

Figure S5. Confocal images of the nanoelectrode array without (a) or with (b) a notch filter in the optical path to filter the reflected light. (c) Time series of fluorescence during a bipolar potential scan from $0.0 \mathrm{v}$ to $1.18 \mathrm{~V}$ with a notch filter to filter the reflection. 